\title{
Environmentally adaptive acoustic transmission loss prediction in turbulent and nonturbulent atmospheres
}

\author{
Gordon Wichern $^{\mathrm{a}}$, Mahmood R. Azimi-Sadjadi ${ }^{\mathrm{a}, *}$, Michael Mungiole $^{\mathrm{b}}$ \\ a Department of Electrical and Computer Engineering, Colorado State University, Fort Collins, CO 80523-1373, USA \\ ${ }^{\mathrm{b}}$ US Army Research Laboratory, Attn: AMSRD-ARL-CI-ES, 2800 Powder Mill Road, Adelphi, MD 20783-1197, USA
}

\begin{abstract}
An environmentally adaptive system for prediction of acoustic transmission loss (TL) in the atmosphere is developed in this paper. This system uses several back propagation neural network predictors, each corresponding to a specific environmental condition. The outputs of the expert predictors are combined using a fuzzy confidence measure and a nonlinear fusion system. Using this prediction methodology the computational intractability of traditional acoustic model-based approaches is eliminated. The proposed TL prediction system is tested on two synthetic acoustic data sets for a wide range of geometrical, source and environmental conditions including both nonturbulent and turbulent atmospheres. Test results of the system showed root mean square (RMS) errors of $1.84 \mathrm{~dB}$ for the nonturbulent and $1.36 \mathrm{~dB}$ for the turbulent conditions, respectively, which are acceptable levels for near real-time performance. Additionally, the environmentally adaptive system demonstrated improved TL prediction accuracy at high frequencies and large values of horizontal separation between source and receiver.
\end{abstract}

(C) 2007 Elsevier Ltd. All rights reserved.

Keywords: Atmospheric acoustics; Turbulent scattering; Parabolic equation; Fuzzy-logic fusion

\section{Introduction}

The problem of modelling and predicting the effects of acoustic waves propagating in the atmosphere has always been of great importance especially in noise forecasting and detection applications (Embleton, 1996). Prediction of acoustic transmission loss (TL), or the diminishment in sound pressure level from a reference point to a receiver depends on a variety of factors, namely problem geometry, source frequency, characteristics of the ground surface, refraction caused by wind and temperature conditions and atmospheric turbulence. Several model-based methods exist to accurately predict TL values in atmospheric acoustic propagation, but the computation times are generally excessive for situations when near real-time environmental awareness is desired. With the increasing importance of Unattended Ground Sensor (UGS) technologies (Srour, 1999) in localizing and tracking acoustic sources, the need for real-time awareness of atmospheric acoustic propagation effects has recently emerged.

\footnotetext{
* Corresponding author. Tel.: +1 970491 7956; fax: +1 9704912249 .

E-mail address: azimi@engr.colostate.edu (M.R. Azimi-Sadjadi).
}

Originally developed for applications in underwater acoustics, several computational methods for modelling acoustic environments have been adapted and successfully applied (Gilbert \& Di, 1993; Salomons, 2001; West, Gilbert, \& Sack, 1992; West, Sack, \& Walden, 1991) to atmospheric acoustic problems over several years. The fast-field program (FFP) (West et al., 1991), Crank-Nicholson parabolic equation (CNPE) (West et al., 1992), and Green's function parabolic equation (GFPE) (Gilbert \& Di, 1993) are among the most extensively studied atmospheric acoustic models. Using any of these techniques, the calculated TL values are generally accepted as reliable (Attenborough et al., 1995), but the computational resources required to compute these TL values is highly variable. The computation times often depend on the maximum vertical and horizontal ranges in which TL values are computed, along with the frequency of the acoustic source. In general, the computation times of the FFP, CNPE, and GFPE methods are simply too long to make them useful for most practical applications. Additionally, inclusion of realistic effects such as atmospheric turbulence or three-dimensional field calculations (Salomons, 2001), further increases the computational intractability of the FFP, CNPE, and GFPE methods. 
Some important aspects of acoustic propagation effects and their influence on source direction-of-arrival (DOA) estimates for passive acoustic arrays operating in atmospheric turbulence are discussed in Wilson (1998). It was reported that the DOA estimation accuracy for a given environment and array configuration tends to degrade in performance as frequency, turbulence strength, and propagation distance between acoustic source and receiver increase. Certain types of atmospheric turbulence, namely those related to the wind velocity were determined to be particularly detrimental to DOA estimation performance accuracy. The work of Wilson (1998) was then modified in Collier and Wilson (2003) and Collier and Wilson (2004) for plane waves and spherical waves, respectively. For simple array geometries (lines and rectangles) the authors showed that atmospheric turbulence is extremely important in calculating accurate performance bounds for acoustic arrays. They speculated that atmospheric turbulence, and possibly other factors such as ground reflections and atmospheric refraction should be included in sensor performance evaluation calculations for all acoustic array geometries.

One problem with methods analyzing performance for acoustic sensors (Collier \& Wilson, 2003, 2004; Wilson, 1998) is the large computational cost inherent in using acoustic prediction methods such as the parabolic equation (PE) model to approximate environmental conditions. In Mungiole and Wilson (2006), the authors attempt to overcome the large computational requirements of traditional acoustic TL calculation methods by using an artificial neural network. Although this neural network-based TL prediction method was found to be promising, inaccurate estimates tend to occur consistently at large values of horizontal separation between source and receiver, large values of source frequency, and during calm, night-time (stable) atmospheric conditions. Although, neural networks have found limited use in atmospheric acoustic applications, they have been extensively utilized in different environmental areas. Following the pioneering work of Chevallier, Chèruy, Scott, and Chedin (1998), where neural networks were used for a weather forecast atmospheric model, the field has grown steadily. For example, in Tang and Hsieh (2003) neural networks assist in the assimilation of surface data into the lower layers of an ocean model. A recent environmental application of neural networks is the hybrid numerical modelling approach of Krasnopolsky and Fox-Rabinovitz (2006), where presented applications include hybrid climate models and a hybrid wind-wave ocean model.

In this paper, a new approach to rapid TL prediction is presented where a different nonlinear neural network prediction model is applied to the data corresponding to a specific environmental condition, thus generating a bank of expert nonlinear predictors. Prior knowledge based on atmospheric physics is used to determine the conditions of interest, and form the foundation of our environmentally adaptive TL prediction system. The environmentally adaptive TL prediction system exploits a decision-making process for selecting the appropriate predictors under a given condition. This process works by assigning a fuzzy confidence (weighting) measure to the output of each predictor in the bank. Furthermore, the decisionmaking device allows the user to embed prior information regarding the likelihood and importance of encountering certain atmospheric and ground conditions. A fusion system is then used to optimally combine the weighted estimates from the different predictors in the bank, obtaining a near real-time TL estimate. In this work we train our neural network-based predictors with data that was synthetically generated using a nondimensional acoustic PE model, and Crank-Nicholson solution scheme (Mungiole \& Wilson, 2006). The synthetic PE model data provides a large, well-controlled and diverse data set for use in neural network generation. The environmentally adaptive prediction system is implemented and benchmarked against the single neural network predictor of Mungiole and Wilson (2006) on the PE model test data. In Wichern, AzimiSadjadi, and Mungiole (2006) the environmentally adaptive TL prediction system was shown to work well when atmospheric turbulence effects were not included. In this work these results are expanded and extensively compared with TL prediction results for a turbulent atmosphere.

This paper is organized as follows: Section 2 provides a brief review of the parabolic equation model for sound propagation in the atmosphere. Section 3 describes the proposed environmentally adaptive TL prediction system. Test results and discussions of the environmentally adaptive system in turbulent and nonturbulent environments are provided in Section 4. Conclusions are presented in Section 5.

\section{Review of the parabolic equation model for sound propagation}

Due to the nonstationary behaviour of the physical atmosphere, as well as the fact that no actual field data set encompasses the variability and randomness required for the large range of parameters used to describe the propagation environment, our data set was synthetically generated using a nondimensional parabolic equation (PE) model and Crank-Nicholson solution scheme (Mungiole \& Wilson, 2006; Salomons, 2001; West et al., 1992). Part of the rationale for using the nondimensional PE model is that it reduces the number of parameters required to specify the propagation environment from 15 or more, to the 10 nondimensional neural network input parameters discussed in Mungiole and Wilson (2006). This dimensionality reduction also improves the accuracy of function approximation (Haykin, 1999). In this section, we briefly describe the main points in deriving the nondimensional PE model; a more thorough derivation is provided in Mungiole and Wilson (2006). The crux of our model as described in West et al. (1992) is the two-dimensional narrow-angle PE, which approximates the full wave equation for atmospheric sound propagation by

$$
\frac{\partial P(x, z)}{\partial x}=\frac{j}{\left(2 k_{0}\right)}\left(\frac{\partial^{2}}{\partial z^{2}}+k_{\mathrm{eff}}^{2}(z)-k_{0}^{2}\right) P(x, z)
$$

where $x$ and $z$ are the horizontal (range) and vertical (elevation) coordinates, respectively, of the sound pressure field in the propagation direction. The reference value of the wave number 
is $k_{0}=2 \pi f / c_{0}$ where $f$ is the frequency and $c_{0}$ is the reference sound speed. We define the effective wavenumber as $k_{\text {eff }}(z)=2 \pi f / c_{\text {eff }}(z)+j v(z)$ where $v$ is an attenuation coefficient modelling the absorption of sound energy as the acoustic wave propagates higher in the atmosphere (Salomons, 2001), and $c_{\text {eff }}$ is the effective sound speed (Wilson, 2003) defined as the speed of sound plus the wind speed component in the propagation direction. The variable $P(x, z)$ represents a substitute for the actual sound pressure denoted by $p(x, z)$, which is obtained from the relation $p(x, z)=$ $P(x, z) \exp \left(j k_{0} x\right) \sqrt{x}$. The cylindrically symmetrical 2-D approximation (West et al., 1992) necessitates the use of this substitute sound pressure. If $\rho_{0}$ represents reference air density, we can define the dimensionless quantities: $\bar{p}=p /\left(\rho_{0} c_{0}^{2}\right), \bar{P}=$ $P \sqrt{\left(c_{0} / f\right)} /\left(\rho_{0} c_{0}^{2}\right), \bar{x}=x f / c_{0}, \bar{z}=z f / c_{0}, \bar{k}_{\mathrm{eff}}=k_{\mathrm{eff}} c_{0} / f$, and $\bar{k}_{0}=k_{0} c_{0} / f=2 \pi$, which result in the nondimensional version of (1) becoming

$\frac{\partial \bar{P}(\bar{x}, \bar{z})}{\partial \bar{x}}=\frac{j}{(4 \pi)}\left(\frac{\partial^{2}}{\partial \bar{z}^{2}}+\bar{k}_{\mathrm{eff}}^{2}(\bar{z})-4 \pi^{2}\right) \bar{P}(\bar{x}, \bar{z})$

where the overbars indicate dimensionless variables.

To compute the acoustic field, the PE model uses a spatial marching procedure where the sound field is calculated at discrete range steps along the propagation path (West et al., 1992). This allows the calculated acoustic field at any given point to incorporate reflection and refraction effects that occurred previously along the propagation path. Additionally, because more refraction is present at high frequencies the step size in the spatial marching procedure is inversely proportional to the source frequency and hence, proportional to wavelength, $\lambda=c_{0} / f$. For the dimensional PE model the step size in both the vertical and horizontal dimensions is $\Delta x=\Delta z=\lambda / 10$, and in nondimensional form, the step size becomes $\Delta \bar{x}=\Delta \bar{z}=1 / 10$, where the frequency information is now embedded in the nondimensional variables, $\bar{x}$ and $\bar{z}$. At low frequencies and small horizontal separations between source and receiver the PE model can compute TL values rather quickly. However, as frequency and horizontal separation increase, the computation time increases dramatically. Furthermore, most acoustic sources of interest contain several different frequency components and require TL calculations at several frequencies making the PE model method intractable in these types of situations. It is for these reasons that we use neural network predictors to approximate the PE model and overcome its inherent computational requirements.

The location of the acoustic receiver can be defined as $\left(\bar{x}_{r}, \bar{z}_{r}\right)$, where $\bar{x}_{r}$ is the non-dimensional horizontal separation between source and receiver and $\bar{z}_{r}$ is the nondimensional receiver height. The sound pressure level (SPL) in decibels $(\mathrm{dB})$ at receiver location $\left(\bar{x}_{r}, \bar{z}_{r}\right)$ can be determined from the following equation (Salomons, 2001):

$\mathrm{SPL}=20 \log _{10}\left|\bar{p}\left(\bar{x}_{r}, \bar{z}_{r}\right)\right|$

where $\bar{p}\left(\bar{x}_{r}, \bar{z}_{r}\right)=\bar{P}\left(\bar{x}_{r}, \bar{z}_{r}\right) \exp \left(j 2 \pi \bar{x}_{r}\right) \sqrt{\bar{x}_{r}}$ is the actual nondimensional sound pressure. Since we are interested in predicting the amount of acoustic attenuation from source to receiver, we refer to SPL diminishment from source to receiver as transmission loss (TL) (Mungiole \& Wilson, 2006) throughout this paper. The PE model output value generated by (3) will be used as the desired value for the training of the neural network predictors.

\subsection{Boundary and initial conditions}

The ground surface causes wave reflections that interact with the direct acoustic wave as it propagates away from the source. The ground surface is assumed flat and incorporated at the bottom of the PE model computational domain using the following boundary condition (Mungiole \& Wilson, 2006):

$$
\frac{\partial \bar{P}_{0}}{\partial \bar{z}}=-j 2 \pi \bar{P}_{0} / \bar{Z}_{c}
$$

where $\bar{P}_{0}=\bar{P}(\bar{x}, 0)$ is the non-dimensional surrogate sound pressure field at the ground surface, and $\bar{Z}_{c}$ denotes the nondimensional ground impedance. The boundary condition in (4) is solved for second order accuracy in this paper. In our formulation we use the model for $\bar{Z}_{c}$ described in Wilson (1997) that is given by

$$
\begin{aligned}
\bar{Z}_{c}= & \bar{q}\left[\left(1+\frac{\gamma-1}{\left(1-j \pi N_{p r} \bar{q}^{2} / \bar{\sigma}\right)^{0.5}}\right)\right. \\
& \left.\times\left(1-\frac{1}{\left(1-j \pi \bar{q}^{2} / \bar{\sigma}\right)^{0.5}}\right)\right]^{-0.5}
\end{aligned}
$$

where $N_{p r}$ and $\gamma$ are the Prandtl Number and the ratio of specific heats for air. The nondimensional static flow resistivity $\bar{\sigma}$, is defined by $\bar{\sigma}=\sigma s_{p}^{2} /\left(f \rho_{0} \Omega\right)$, where $s_{p}$ is the pore shape factor, and $\Omega$ is the porosity (Attenborough, 1985). The tortuosity (Attenborough, 1985) to porosity ratio $\bar{q}$ is defined as $\bar{q}=q / \Omega$, where $q$ is the tortuosity. The static flow resistivity $\sigma$ models the hardness of the ground surface with high values corresponding to hard ground (e.g. asphalt) and low values corresponding to soft ground (e.g. fresh snow). The porosity $\Omega$ models the number of pores in the ground surface, and the tortuosity $q$ models the twisting of the pores. The pore shape factor, porosity and tortuosity are all unitless parameters.

The computational grid used in the nondimensional PE model is typically truncated at a certain height. This causes computationally spurious reflections to occur at the height where the PE model grid is truncated. Note that these reflections are not present in the actual physical environments, as the actual atmosphere does not have a well-defined "boundary". To minimize the effects of computationally spurious reflections at the top of the grid, we construct an absorbing layer according to the model in Mungiole and Wilson (2006).

The initial condition from which the finite difference solution of the PE is spatially marched forward (sound pressure at $\bar{x}=0$ ) is the Gaussian starter function from Mungiole and Wilson (2006) that depends on the nondimensional source strength, $\bar{S}=S / \rho_{0} c_{0}^{2}$, the nondimensional source height, $\overline{z_{s}}=$ $z_{s} f / c_{0}$, and the surface reflection coefficient $R=\frac{\bar{Z}_{c}-1}{\bar{Z}_{c}+1}$ where $\bar{Z}_{c}$ is the nondimensional ground impedance defined in (5). 


\subsection{Atmospheric profile}

We now consider the effect of refraction, which is the bending of sound waves in the near ground atmosphere due to vertical gradients in wind velocity and temperature, both of which directly relate to the speed of sound. Different atmospheric profiles can cause sound to refract in different directions, and hence cause hot spots (regions where sound levels are locally elevated) and shadow regions (where sound levels are locally depressed) to appear in different locations of the sound field. The near ground atmospheric profiles used in our study fall from the equation for the effective sound speed (Wilson, 2003)

$c_{\text {eff }}(z)=c(z)+u(z) \cos \beta$

where $u(z)$ is the wind component in the horizontal direction, $\beta$ is the azimuthal angle (angle between the sound propagation and wind directions), and $c(z)$ is the actual sound speed in air described by

$c(z)=c_{0}\left(1+\frac{T^{\prime}(z)}{2 T_{0}}\right)$.

Here, $T^{\prime}$ symbolizes the quantity of a small temperature variation about the ambient value $T_{0}$. It is assumed, for this formulation of the sound speed $c$, that the effect of humidity is small and hence negligible. The near ground temperature $T$ and wind velocity $u$ profiles (the latter of which is also influenced by the ground roughness length $z_{0}$ ) are formed using the Monin-Obukhov similarity theory (Monin \& Obukhov, 1954), and derived in Mungiole and Wilson (2006). From (6) and (7) we see that the wind and temperature profiles essentially describe the effective sound speed as a function of height. For a given atmospheric condition, the temperature gradient is $T^{\prime}(z)=T(z)-T\left(z_{\text {ref }}\right)$, where $z_{\text {ref }}$ is a reference height ( $z_{\text {ref }}=5 \mathrm{~m}$ for our experiments). This temperature gradient can essentially be described by the sound speed fluctuation scale parameter, $c_{*}$ (Wilson, 2003), which can be written as $c_{*}=$ $c_{0} T_{*} /\left(2 T_{0}\right)$, where $T_{*}$ is a temperature scale representing the covariance of vertical velocity and temperature at the surface. Similarly, the wind speed profile as a function of height $u(z)$ can essentially be described by the friction velocity, denoted by $u_{*}$. Details on the exact formulations of $T^{\prime}(z)$ and $u(z)$ used in this work can be found in Monin and Obukhov (1954) and Mungiole and Wilson (2006), but in the following we are only concerned with the values of $c_{*}$ and $u_{*}$ for a given atmospheric state. The nondimensional versions of $c_{*}$ and $u_{*}$ are given by $\bar{c}_{*}=c_{*} / c_{0}$ and $\bar{u}_{*}=u_{*} / c_{0}$, respectively. The ratio of adiabatic coefficients for sound speed and wind is denoted by $A$ (Wilson, 2003), and equals the temperature dependent sound speed scale $c_{*}$ divided by the wind dependent friction velocity $u_{*}$, i.e., $A=c_{*} / u_{*}$. This ratio is important in governing the direction of sound refraction in the atmosphere, and illustrates the relative contributions of wind and temperature gradients in the acoustic atmosphere as they relate to sound refraction. Typically, values of $A>0$ occur during calm nighttime conditions, and cause sound to refract downward. We will refer to this condition as the stable atmospheric regime (Stull, 1988).
Atmospheric conditions when $A<0$ tend to occur when the sun heats the ground during the daytime, and cause sound to be refracted upward. We will refer to this condition as the unstable atmospheric regime (Stull, 1988). Since the windrelated friction velocity term $u_{*}$ is always positive, the sign of the sound speed fluctuation scale $c_{*}$ is the determining factor between the stable and unstable regimes.

\subsection{Model parameters as inputs to TL predictors}

As our ultimate goal is to approximate the acoustic propagation solution of the PE model using neural networks for improved prediction speed, we must define a parameter set to be used as neural network inputs. We now discuss the 10 parameters that are used as neural network inputs, and also specify the acoustic environment simulated by the PE model. To select the range of these individual input parameters, we choose values that cover most atmospheric and ground conditions likely to be encountered in practice, as well as frequency and geometrical ranges that are of tactical importance in UGS acoustic environments. Further justification of the parameter ranges is provided in Mungiole and Wilson (2006).

A number of parameters can be eliminated from consideration since they do not affect the sound pressure level (Mungiole \& Wilson, 2006). These include the nondimensional source strength $\bar{S}$, which can be eliminated because we are interested in predicting TL values, not the actual sound pressure. In addition, two absorbing layer parameters, the attenuation coefficient and absorbing layer depth, can also be eliminated if we presume that this layer controls sound reflections. Finally, we specify that a constant temperature reference height is used for each synthetic case.

Table 1 provides the range of values used in our study as well as the baseline and extreme values for each input parameter. The baseline values are likely to be encountered in practice, and are often near the middle of the range for each parameter. The extreme parameter values, primarily for the parameters that strongly influence TL (Mungiole \& Wilson, 2006), are generally selected to be close to one end of the range of the respective parameter. If the baseline value was near the end of the range, the extreme value was at or near the opposite end of the range of the respective parameter. Extreme parameter values will be used in this study to examine the robustness of the developed prediction systems.

The combination of the sound speed fluctuation scale $c_{*}$ and friction velocity $u_{*}$ allow coverage between the unstable and weakly stable atmospheric regimes, i.e. both calm and windy conditions during the daytime and windy conditions during the night-time. Additionally, the meteorological parameters $c_{*}$ and $u_{*}$ have their values constrained by the limitations of the Monin-Obukhov similarity theory (Monin \& Obukhov, 1954), which breaks down in the highly stable atmosphere when $A \gg$ 0 . To avoid such cases in our synthetic data set, a filter is implemented (Mungiole, Wilson, \& Ostashev, 2004), based on calculated values of the surface heat flux, and inverse Obukhov length. Both of these parameters are functions of $c_{*}$ and $u_{*}$ only, 
Table 1

Range and baseline parameter values used to specify the PE model data set in our study

\begin{tabular}{|c|c|c|c|c|c|}
\hline Parameter (units) & Non-dimensional definition & Min & $\operatorname{Max}$ & Baseline & Extreme \\
\hline Frequency $(\mathrm{Hz})$ & N/A & 20 & 200 & 50 & 200 \\
\hline Horizontal separation (m) & $\bar{x}_{r}=x_{r} f / c_{0}$ & 100 & 900 & 500 & 900 \\
\hline Source height (m) & $\bar{z}_{s}=z_{s} f / c_{0}$ & 0 & 5 & 0 & 5 \\
\hline Receiver height (m) & $\bar{z}_{r}=z_{r} f / c_{0}$ & 0 & 5 & 0 & 5 \\
\hline Azimuthal angle (rad) & $\beta$ & 0 & $\pi$ & 0 & $\pi$ \\
\hline Friction velocity $(\mathrm{m} / \mathrm{s})$ & $\bar{u}_{*}=u_{*} / c_{0}$ & 0.05 & 0.5 & 0.28 & 0.067 \\
\hline Sound-speed scale $(\mathrm{m} / \mathrm{s})$ & $\bar{c}_{*}=c_{*} / c_{0}$ & -1.18 & 0.1 & -0.2 & -1.11 \\
\hline Static flow resistivity $(\mathrm{Pa} \mathrm{s} / \mathrm{m}) \times 10^{6}$ & $\bar{\sigma}=\sigma s_{p}^{2} /\left(f \rho_{0} \Omega\right)$ & 0.025 & 2 & 0.2 & 1 \\
\hline Tortuosity/porosity & $\bar{q}=q / \Omega$ & 1.54 & 10 & 2.8 & 7.5 \\
\hline Roughness length (m) & $\bar{z}_{0}=z_{0} g / c_{0}^{2}$ & 0.001 & 0.1 & 0.01 & 0.01 \\
\hline
\end{tabular}
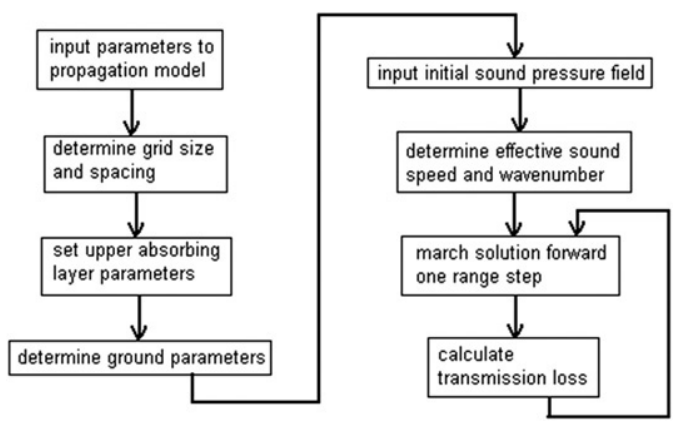

Fig. 1. Schematic of the PE model TL computation procedure.

and the filter eliminates any case for which the combination of $c_{*}$ and $u_{*}$ violates the theory.

A schematic identifying the main steps involved in executing the sound propagation model is given in Fig. 1. This schematic indicates that the specified parameters are input to the model followed by determining the grid to be used. Some initial and boundary conditions are then set and the effective sound speed and wave number are determined for the entire grid. After these preliminary steps are completed, the marching procedure is performed during which the SPL and TL are obtained for each vertical grid location at the next horizontal grid location. SPL and TL values are obtained for each successive horizontal grid location until the particular horizontal separation value is reached. The TL at the combination horizontal and vertical grid location for the particular case being simulated is then saved as the target value required for neural network development.

The nondimensional PE model just described will be used to create a data set, which will subsequently be used to train neural network predictors to capture the input-output mapping between the input parameters used to describe the PE model and the TL value generated by the PE model. The neural network input data is defined using the 10 parameters from Table 1 as $\mathbf{x}=\left[f, \bar{x}_{r}, \bar{z}_{s}, \bar{z}_{r}, \beta, \bar{u}_{*}, \bar{c}_{*}, \bar{\sigma}, \bar{q}, \bar{z}_{0}\right]^{\mathrm{T}}$, where each component of $\mathbf{x}$ is varied independently and uniformly over its specified range. Due to the Monin-Obukhov similarity theory (Monin \& Obukhov, 1954) constraints in the stable atmospheric regime discussed above, the parameters $c_{*}$ and $u_{*}$ have slightly nonuniform distributions. The output of the PE model from (3) is defined as $y$, and used as the desired value in neural network training. Once trained, our neural network-based predictors are expected to accurately approximate the PE model in all geometrical, atmospheric and ground conditions of interest over a range of frequencies without the high computational cost inherent in the PE model.

\subsection{Turbulent scattering}

Up to this point, our discussions on the PE model concentrated on a nonturbulent atmosphere, where the effective sound speed profile is smooth (e.g. logarithmic). As discussed in Salomons (2001) this profile should be thought of as an average profile, where the effective sound speed as a function of height has been averaged over an approximately $10 \mathrm{~min}$ long interval. In reality, atmospheric turbulence causes the temperature and wind profiles (used to define the effective sound speed) to vary considerably on time scales of seconds or minutes, and the instantaneous effective sound speed profile is not a smooth function of height. The effect of atmospheric turbulence varies depending on how the wind and temperature gradients shape the effective sound speed profile. In an upward refracting (unstable) atmosphere refractive shadow regions are present near the ground due to the upward bending of sound waves. Without turbulence upward refraction causes all sound waves to bend above these shadow regions leading to very low sound pressure values. The addition of atmospheric turbulence causes large increases in the sound pressure levels usually present in an acoustic shadow region, as the random turbulent fluctuations cause slight changes to the direction of propagation. These changes in propagation direction allow more sound to penetrate into a shadow region and are often referred to as turbulent scattering. In a downward refracting (stable) atmosphere, turbulence also causes an increase in the sound pressure level, but for different reasons. Downward refraction causes a large number of reflected sound waves, and when the direct and reflected waves are $180^{\circ}$ out of phase, interference minima occur leading to a sharp reduction in sound pressure. The inclusion of turbulence in essence leads to random phase fluctuations of the direct and reflected waves, which generally reduce the depth of the interference minima (Salomons, 2001).

The method used to simulate atmospheric turbulence for the PE model data set is described in Wilson (2000). This method, which uses empirical orthogonal functions (Lumley, 1971) to generate a turbulence field, effectively works by 
adding a random phase fluctuation at each range step of the PE model algorithm. In this paper, the simulated turbulence fields had a resolution of $0.45 \mathrm{~m}$ and a height of $1 / 10$ the horizontal separation, thus, the turbulence height would range between 10 and $90 \mathrm{~m}$ using the $x_{r}$ values from Table 1. Adding turbulence to the PE model introduces a certain amount of randomness, meaning that for a given combination of input parameters two different runs of the turbulent PE model will yield two different TL values. Thus, errors could be due to a combination of random turbulent fluctuations and inaccuracies in the neural network mapping function. To better assess the errors of the neural network mapping function, we obtain an ensemble average TL value from the turbulent PE model by performing 50 realizations for a given combination of input parameters, which is considered as the output of the turbulent PE model. The turbulent PE model provides a more realistic TL value than the nonturbulent model, but simulating the turbulence field for a single realization requires approximately twice the computer processing time to compute the TL values. Also, because 50 realizations are required to generate a single average TL value, it takes approximately 100 times longer to obtain a TL value from the turbulent PE model as opposed to the nonturbulent one. The increased computational requirements of the turbulent PE model make the development of fast methods for TL prediction even more important.

\section{An environmentally adaptive TL prediction system}

Fig. 2 shows the block diagram of the proposed environmentally adaptive TL prediction method. In this figure, $\mathbf{x}_{j}$ represents the $j$ th input vector, $\hat{y}_{i j}$ is the estimate from the $i$ th predictor in the bank, $C_{i j}$ is the confidence measure given to the $i$ th predictor in the bank, and $\hat{y}_{T L_{j}}$ is the final output of the system. The environmentally adaptive system provides improved acoustic TL prediction when compared to a single neural network, which cannot accurately handle the drastic environmental changes caused by the high variability of the input parameters in the PE model data sets. This structure also provides the flexibility to modify the prediction system if new environmental conditions are encountered, while providing the ability to fine tune the system for increased accuracy under conditions of interest.

Each back-propagation neural network (BPNN) predictor in Fig. 2 is trained for a specific environmental condition corresponding to a specific combination of selected input parameters. The prediction results of these BPNNs are given fuzzy confidence measures (close to one for the most appropriate predictor, or close to zero for the least appropriate), and then combined using another BPNN in the fusion centre. It is envisaged that this mixture of environmentally specific experts will improve overall acoustic TL prediction accuracy using the principle of divide and conquer (Haykin, 1999).

\subsection{Environmentally specific data subsets}

In order to create expert networks for specific environmental conditions we must first partition the data into environmentally

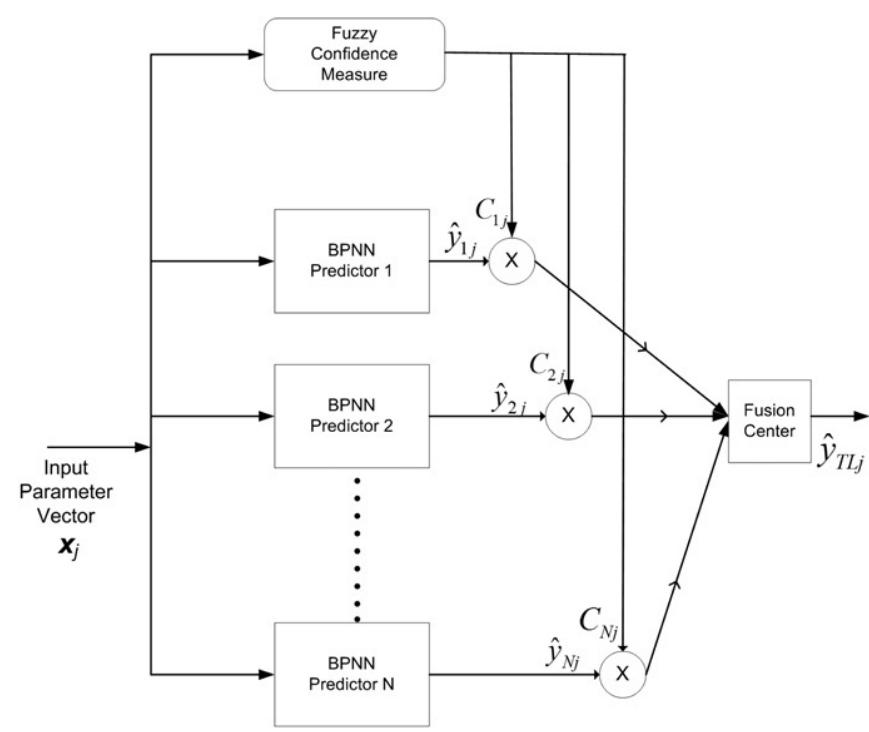

Fig. 2. Block diagram of the environmentally adaptive TL prediction system.

specific data sets. Traditionally, an unsupervised learning technique, e.g. clustering (Hastie, Tibshirani, \& Friedman, 2001) is used to partition input data into various subsets. In our application we have prior knowledge of the physical mechanisms responsible for the variation in TL values generated by the PE model in different regions of the input space. Since this knowledge is available, we will use it to partition the data into environmentally specific subsets as opposed to an unsupervised learning algorithm.

The number of environmentally specific data subsets will be signified by $L$. These environmentally specific data subsets denoted by $D_{i}, i=1, \ldots, L$ correspond to specific environmental conditions from the data set (i.e. high $c_{*}$ values, low $u_{*}$ values, etc.). The $D_{i}$ have soft membership meaning that a given input sample $\mathbf{x}_{j}$ can belong to multiple environmentally specific data subsets (e.g. sample $\mathbf{x}_{j}$ can have a low $c_{*}$ value and a high $u_{*}$ value).

For the PE model data we partition the available cases into six environmentally specific data subsets $(L=6)$. We chose six data subsets, because using fewer would not allow significant partitioning and the prediction scheme would exhibit behaviour similar to a single BPNN. If more than six environmentally specific data subsets were used there would not have been sufficient data to train predictors for all conditions of interest. Another factor to be considered when partitioning the data is the inclusion of overlap between environmentally specific data subsets. Having samples belong to multiple environmentally specific data subsets allows multiple experts to contribute in estimating the output TL.

For the presented data-partitioning method, the parameters chosen to divide the original data set were the friction velocity $u_{*}$, and the sound speed fluctuation scale $c_{*}$. These parameters were chosen because the results in Mungiole and Wilson (2006) showed that they are environmental parameters with a significant influence on the TL, and also responsible for increased neural network output errors as they were varied over their respective ranges. The combination of $c_{*}$ (temperature) 
and $u_{*}$ (wind) govern the direction sound waves are refracted as they propagate higher in the atmosphere. Thus, each partitioned data subset can be thought of as corresponding to different atmospheric refraction conditions. Note that other important parameters such as frequency, $f$, and horizontal separation between source and receiver, $x_{r}$, were not chosen as they are dependent on the source of interest and not the environment.

The six environmentally specific conditions used in our partitioning method are as follows:

(1) High values of $u_{*}\left(u_{*} \geq 0.275 \mathrm{~m} / \mathrm{s}\right)$;

(2) High values of $c_{*}\left(c_{*} \geq 0 \mathrm{~m} / \mathrm{s}\right)$;

(3) Moderate values of $u_{*}\left(0.125 \mathrm{~m} / \mathrm{s}<u_{*}<0.425 \mathrm{~m} / \mathrm{s}\right)$;

(4) Moderate values of $c_{*}\left(c_{*} \geq-0.9 \mathrm{~m} / \mathrm{s}\right)$;

(5) Low values of $u_{*}\left(u_{*}<0.275 \mathrm{~m} / \mathrm{s}\right)$;

(6) Low values of $c_{*}\left(c_{*}<-0.3 \mathrm{~m} / \mathrm{s}\right)$.

The high, medium and low $u_{*}$ data subsets were chosen to divide the entire range of $u_{*}$ into roughly equal thirds with significant portions of overlap. The high $c_{*}$ subset $\left(c_{*}>0\right)$ was chosen because it corresponds to the stable atmospheric regime and downward refraction conditions where the PE model behaviour is highly variable as a function of $c_{*}$. The medium and low $c_{*}$ data subsets were chosen to partition the entire range of $c_{*}$ into approximately equal halves with significant portions of overlap. Partitioning of the environmentally specific data sets using parameters besides $c_{*}$ and $u_{*}$, namely $c_{*}$ and the ground-related static flow resistivity $\sigma$ was explored in Wichern (2006). In terms of the performance metrics discussed in Section 4 the overall difference between the partitioning schemes was less than $2 \%$, with the presented partitioning method (based on $c_{*}$ and $u_{*}$ ) tending to provide slightly better TL prediction performance for the data sets examined in this paper. When comparing different partitioning methods, the alternative ground-based partitioning scheme (based on $c_{*}$ and $\sigma$ ) improved performance under soft ground conditions, while sacrificing TL prediction accuracy in the highly unstable atmospheric regime. Once these environmentally specific data subsets are partitioned, they are used to train the environmentally specific predictors shown in Fig. 2.

\subsection{Environmentally specific predictors}

In order to train the environmentally specific BPNNbased predictors of Fig. 2 on the partitioned data subsets described in Section 3.1, we first randomly subdivided our PE model data sets into thirds forming training, validation and testing sets (prior to partitioning of the data into the environmentally specific data subsets described in Section 3.1). The purpose of the validation set is to select the best-trained networks for different topologies, training algorithms and weight initializations. The testing set is used for performance evaluation of the selected BPNN's on data they have never seen before. Note that although the training and validation sets are different for each expert predictor, the testing set is the same for all the environmentally specific predictors in Fig. 2. That is, each environmentally specific BPNN produces a prediction of the TL for a given combination of input parameters.
Next, we need to choose appropriate network structures and training algorithms. Two- and three-layer BPNN predictors with varying number of hidden-layer neurons were tried to find the optimum network structures. For each environmentally specific predictor in Fig. 2, except the one for the $c_{*}>0$ subset, a two-layer network with 10-45-1 structure, i.e. 10 inputs (10 dimensional vector $\mathbf{x}_{j}$ ), 45 hidden layer neurons and one output (estimated TL $\hat{y}_{j}$ ), was found to provide the best overall results. Note that this is consistent with the findings in Mungiole and Wilson (2006). To find this structure the number of hidden layer neurons was varied from 15 to 60 in steps of 15 and the trained networks were then tested on the validation data sets. For the $c_{*}>0$ subset, a three-layer BPNN with structure 10-22-111 had to be used in order to obtain acceptable TL prediction results. Again, this structure was arrived at experimentally by varying the number of first and second hidden-layer neurons in the ranges $20-25$ and $10-15$, respectively. For the two-layer BPNN predictors, the selected training algorithm was based upon the fast Levenberg-Marquardt (LM) algorithm (Hagan \& Menhaj, 1994), For the three-layer BPNN, a traditional gradient algorithm (Haykin, 1999) with an adaptive learning rate and momentum constant of 0.9 , was more effective than the LM training method. For each expert network, 10 different random weight initializations were tried, and the one that led to the best performance on the validation set was chosen and subsequently used for further testing.

\subsection{Confidence measure assignment}

The assignment of the confidence measure allows for incorporation of prior knowledge of the problem domain or some user information, which is not typically present in the training data, to be included in the prediction system. Thus, the confidence measure component is treated independently from the environmentally specific predictors and the fusion centre, whose parameter values depend only on the training data. The purpose is to assign confidence weights to the output of each predictor in Fig. 2, depending on the employed partitioning method, the value of the input parameter vector, $\mathbf{x}_{j}$, and other prior environmental and operational information. This confidence measure assignment can be accomplished using fuzzy set theory (Zadeh, 1965), which can determine how appropriate each environmentally specific predictor is for a given input vector. This is due to the fact that fuzzy sets allow natural language terms such as high, medium, and low to be represented mathematically, by allowing elements to be partial members of different sets with varying degrees of membership at the same time (Harris, Hong, \& Gan, 2002).

Here, we provide a brief review of fuzzy measures for the purpose of clarity. For a detailed treatment the reader is referred to Harris et al. (2002), Lin and Lee (1991), and Klir and Yuan (1995). A fuzzy variable (Harris et al., 2002) $V_{z}$ is described by a 4-tuple: $\{z, T(z), \chi, M(z)\}$ where $z$ is the symbolic name or linguistic variable, $T(z)=\left\{T_{z}^{1}, T_{z}^{2}, \ldots, T_{z}^{L}\right\}$ is the set of linguistic labels associated with $z, \chi$ is a closed interval of real numbers containing the range of values over which $T(z)$ is defined, and $M(z)=\left\{M_{z}^{1}, M_{z}^{2}, \ldots, M_{z}^{L}\right\}$ is a semantic 
rule function that returns the meaning of a given linguistic label in terms of its membership function. That is, $T_{z}^{\ell}, \ell=$ $1,2, \ldots, L$ is a linguistic label associated with membership function $M_{z}^{\ell}, \ell=1,2, \ldots, L$. For example, if linguistic variable $z$ represents wind speed, then $T(z)$ might be sslow, medium, fast $\}, \chi$ may be $[0,100]$ (miles per hour), and $M(z)$ represents the membership functions assigned to each element in $T(z)$.

Given the $j$ th input vector, $\mathbf{x}_{j}$, and its predicted output value from the $i$ th predictor in the bank $\hat{y}_{i j}$, we would like to assign a confidence measure $C_{i j}$ to $\hat{y}_{i j}$ based upon the degree of membership that input sample $\mathbf{x}_{j}$ belongs to the $i$ th environmentally specific data set, $D_{i}$. This is done by defining a fuzzy variable for each input parameter that is used to partition the input data into environmentally specific subsets following the procedure of Section 3.1. If we let $z_{k}$ denote the $k$ th parameter used to partition the data (i.e. $c_{*}$ or $u_{*}$, in this case) we define the fuzzy variables $V_{z_{k}}$, as

$$
\begin{aligned}
& V_{z_{k}}=\left\{z_{k},\left\{T_{z_{k}}^{1}, T_{z_{k}}^{2}, \ldots, T_{z_{k}}^{L_{k}}\right\}, \chi_{k},\left\{M_{z_{k}}^{1}, M_{z_{k}}^{2}, \ldots, M_{z_{k}}^{L_{k}}\right\}\right\}, \\
& \quad \text { for } k=1,2, \ldots, K
\end{aligned}
$$

where $K$ is the number of parameters namely $c_{*}$ and $u_{*}, \chi_{k}$ is the range of real numbers associated with $z_{k}, T_{z_{k}}^{\ell_{k}}, \ell_{k}=$ $1,2, \ldots, L_{k}$ is the linguistic label associated with membership function $M_{z_{k}}^{\ell_{k}}$, and $L_{k}$ is the number of fuzzy sets affiliated with $z_{k}$. For the $j$ th input vector $\mathbf{x}_{j}$ the value of the $k$ th partitioning parameter $z_{k}(j)$, is mapped to the fuzzy set labeled by $T_{z_{k}}^{1}$ with membership degree $M_{z_{k}}^{1}\left(z_{k}(j)\right)$ and to the fuzzy set labelled by $T_{z_{k}}^{2}$ with membership degree $M_{z_{k}}^{2}\left(z_{k}(j)\right)$, and so on. For example, in this data-partitioning method $z_{1}=c_{*}$, $z_{2}=u_{*}, T_{z_{1}}=\left\{\right.$ High $c_{*}$, Medium $c_{*}$, Low $\left.c_{*}\right\}, T_{z_{2}}=$ $\left\{\right.$ High $u_{*}$, Medium $u_{*}$, Low $\left.u_{*}\right\}, \chi_{1}=[-1.18,0.1](\mathrm{m} / \mathrm{s})$, and $\chi_{2}=[0.05,0.5](\mathrm{m} / \mathrm{s})$, where the values of $\chi_{k}$ cover the entire range of $c_{*}$ and $u_{*}$ from Table 1 . The sets of membership functions $\left\{M_{z_{1}}^{1}, M_{z_{1}}^{2}, M_{z_{1}}^{3}\right\}$ and $\left\{M_{z_{2}}^{1}, M_{z_{2}}^{2}, M_{z_{2}}^{3}\right\}$ for this partitioning method are shown in Fig. 3(a) and (b) for $z_{1}=c_{*}$ and $z_{2}=u_{*}$, respectively. Fig. 3(a) and (b) show the adopted Gaussian membership functions (Harris et al., 2002), $M_{z_{k}}^{\ell_{k}}\left(z_{k}(j)\right)$, with mean and variance $\hat{m}_{\ell_{k}}$ and $\hat{\sigma}_{\ell_{k}}^{2}$ that are computed using all the training samples belonging to $D_{i}$. Note that this Gaussian membership function is not a pdf, rather it gives a degree of membership that a given value satisfies a fuzzy concept. As discussed in Harris et al. (2002), there is no unique fuzzy membership function for a given problem. Besides the Gaussian there are several other common fuzzy membership functions (Klir \& Yuan, 1995), such as triangular or $B$-spline. Our use of the Gaussian membership function is motivated by several reasons: (1) computational tractability, (2) smooth and local behaviour, and (3) allow overlap between the different predictors in the bank for generating multiple predictions with different confidence measures. The confidence measure $C_{i j}$ assigned to each predicted output $\hat{y}_{i j}$, based upon the degree of membership that input sample $\mathbf{x}_{j}$ belongs to the $i$ th environmentally specific data set, $D_{i}$ can be determined from the membership function using $C_{i j}=C_{\left(k \ell_{k}\right) j}=M_{z_{k}}^{\ell_{k}}\left(z_{k}(j)\right)$ where $j$ is the input sample index, in our application $k=1,2$ is the index of our parameter of interest (i.e., $z_{1}=c_{*}, z_{2}=u_{*}$ ), and $\ell_{k}=1,2,3$ is the index of the fuzzy set (i.e. $\ell=1, k=1$ corresponds to the High $c_{*}$ set, $\ell=2, k=1$ corresponds to the Medium $c_{*}$ set, and so on). For notational convenience the index $i$ represents a relabeling of the double index $\left(k \ell_{k}\right)$, i.e. $i=L_{k}(k-1)+\ell_{k}$. In our application, we also find it convenient to normalize the $C_{i j}$ for a given sample $j$, and add a term that allows for prior weighting of each environmentally specific data subset $D_{i}$. This is done by redefining $C_{i j}$ as

$C_{i j}=\frac{\alpha_{i} C_{i j}}{\sum_{i=1}^{M} \alpha_{i} C_{i j}}$

where $\alpha_{i}$ is a prior weighting term assigned to each expert, and represents a prior knowledge that an input sample belongs to $D_{i}$, and/or that the user is more confident in the $i$ th predictor of the bank. This provision provides the ability to heavily weight conditions of interest. For example, if the environmentally adaptive TL prediction system was operating in an environment where calm wind conditions were very likely to occur (low $u_{*}$ values), the user could imbed a high value for $\alpha_{i}$, corresponding to the low $u_{*}$ data set, and help to offset possible inaccuracies in the monitoring equipment used to measure the environmental parameters. If for some reason certain predictors are known to give unreliable estimates due to modelling or measurement errors while generating their training data, this information can also be embedded into the $\alpha_{i}$ terms. For the experiments presented here we assume that all $D_{i}$ are equally reliable and likely to be encountered in practice, i.e. $\alpha_{i}=1$ for all $i$, in our presented results.

The environmentally specific predictor outputs, $\hat{y}_{i j}$ are multiplied by their corresponding confidence measure $C_{i j}$, and used to form the vector of weighted predictions $\mathbf{b}_{j}=$ $\left[C_{1 j} \hat{y}_{1 j}, C_{2 j} \hat{y}_{2 j}, \ldots, C_{L j} \hat{y}_{L j}\right]^{\mathrm{T}}$. The vector $\mathbf{b}_{j}$ containing the weighted estimates of the $L$ environmentally specific predictors is then applied to the fusion centre for sample $\mathbf{x}_{j}$.

\subsection{Fusion centre design}

The combination rule employed by the fusion centre in Fig. 2 can take either a linear or nonlinear approach (Benediktsson, Sveinsson, \& Swain, 1997). Here, we used a nonlinear fusion system using a separate BPNN. Assuming $n$ available input parameter vectors in the training set, the weighted estimates of the $L$ environmentally specific predictors are used to form the $n \times L$ matrix $\mathbf{B}=\left[\mathbf{b}_{1}, \mathbf{b}_{2}, \ldots, \mathbf{b}_{n}\right]^{\mathrm{T}}$ of weighted estimate vectors, $\mathbf{b}_{j}$ 's. We denote the desired output vector (TL values generated by the PE model) of size $n \times 1$ as $\mathbf{y}=$ $\left[y_{1}, y_{2}, \ldots, y_{n}\right]$. The input data matrix $\mathbf{B}$ and desired vector $\mathbf{y}$ are then used as the training data for the fusion BPNN. For our TL prediction problem, a BPNN of structure 6-30-1, where the six inputs correspond to the weighted estimates from the ( $L=6$ ) environmentally specific predictors, $\mathbf{b}_{j}$, was found to provide the best results. The final output of the environmentally adaptive system in Fig. 2 for the $j$ th training sample, which is defined by $\hat{y}_{T L_{j}}$, can be obtained from the value of the single (linear) output neuron of this fusion network. The final TL 


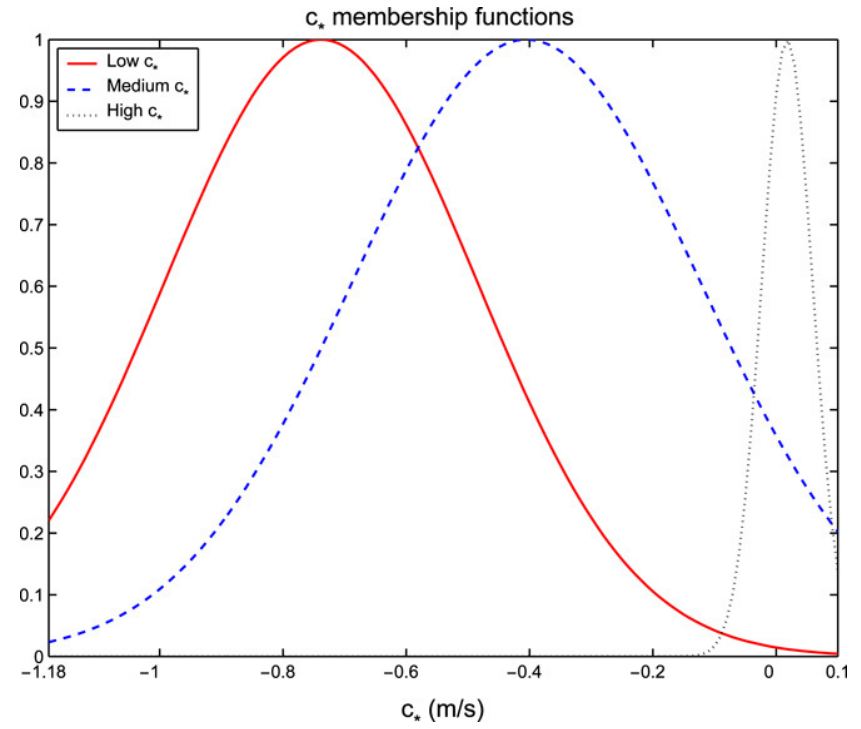

(a) $\left\{M_{z_{k}}^{1}, M_{z_{k}}^{2}, M_{z_{k}}^{3}\right\}$, for $z_{k}=c_{*}$.

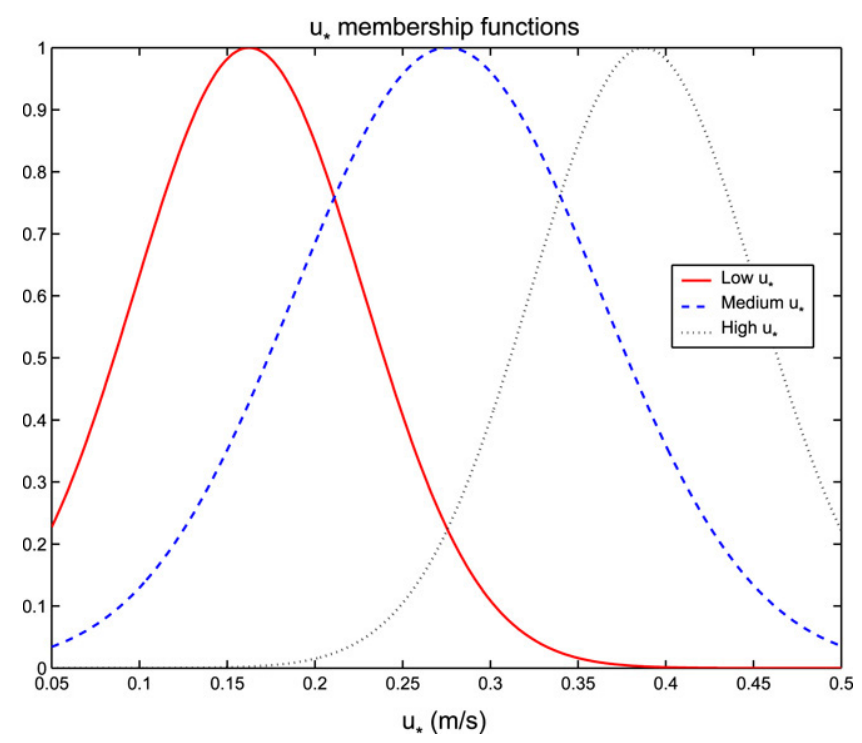

(b) $\left\{M_{z_{k}}^{1}, M_{z_{k}}^{2}, M_{z_{k}}^{3}\right\}$, for $z_{k}=u_{*}$.

Fig. 3. Membership functions used for the data-partitioning method based on $c_{*}$ and $u_{*}$.

prediction $\hat{y}_{T L_{j}}$, is then compared with the actual PE model output (SPL from Eq. (3)) to determine the performance of the environmentally adaptive prediction system.

\section{Results and discussion}

\subsection{Environmentally adaptive TL prediction accuracy}

In evaluating the performance of the environmentally adaptive prediction system presented in Section 3 we benchmark its performance against the single BPNN prediction method discussed in Mungiole and Wilson (2006) on the actual $\mathrm{PE}$ model test data. The performance measures used to evaluate TL prediction accuracy are the root mean square (RMS) error criterion computed over all cases in the data set. The other performance measure used in our experiments is the percentage of cases where the magnitude of the error on the testing set was greater than a $2 \mathrm{~dB}$ threshold value, which is representative of the percentage of unreliable estimates (outliers). In addition to comparing the quantitative performance measures of the two methods it is also important to note that the environmentally adaptive system provides the flexibility to incorporate prior knowledge into the system, and that its modular architecture can be easily modified when new conditions are encountered.

Using the nondimensional PE model, a data set of $N=$ 40, 163 pairs were generated for the nonturbulent atmospheric condition, and $N=25,204$ pairs for the turbulent condition. With each pair consisting of an input parameter combination and its associated TL value, i.e. $\left\{\mathbf{x}_{j}, y_{j}\right\}_{j=1}^{N}$. Each data set (nonturbulent or turbulent) was then divided into thirds forming the training, validation and testing sets used to build and evaluate the neural network-based prediction systems. The reason for the smaller size of the turbulent data set is due to the increased computation time required to generate a single case as discussed in Section 2.4. Table 2 gives a performance measure comparison of the environmentally adaptive and single BPNN prediction methods for both the non-turbulent and turbulent conditions. As can be observed, the environmentally adaptive method reduces the RMS error on the testing set by $15.6 \%$ for the nonturbulent condition, and $12.3 \%$ for the turbulent condition. We also notice a greater increase in RMS error values from the training to the testing sets for the turbulent condition in Table 2, when compared with the nonturbulent RMS error values. This may be attributed to the smaller size of the turbulent data set when compared to that of the nonturbulent data set. As a result, it is possible that certain regions of the 10-dimensional input space are not covered in the training set, hence leading to the poor generalization in those regions. Additionally, it is important to note that the environmentally adaptive method reduces the percentage of cases in the testing set where the magnitude of error is greater than $2 \mathrm{~dB}$ (outliers) by $24.1 \%$ for the nonturbulent condition and $27.5 \%$ for the turbulent condition, when compared to the single BPNN prediction method of Mungiole and Wilson (2006). The results lead to the important observation that while the environmentally adaptive system is effective in reducing the overall RMS error, it performs proportionately better on outliers when compared to the single BPNN. This reduction in the number of outliers is extremely important, as it demonstrates that the environmentally adaptive prediction system is much more likely to estimate a reliable TL value, which is within $2 \mathrm{~dB}$ of the value calculated using the PE model. It is evident in Table 2 that the turbulent atmospheric condition has reduced RMS errors and number of outliers, when compared to the nonturbulent atmosphere. As previously indicated, each turbulent synthetic case represents the average TL obtained for an ensemble of 50 realizations of the same combination of input parameters. Most of these ensemble average cases resulted in reduced errors because they tended to smooth out the sound pressure level field by increasing the energy that scatters into shadow zones and decreasing the energy entering hot spots, 
Table 2

RMS error comparison of environmentally adaptive and single BPNN prediction methods on the PE model data set

\begin{tabular}{lllll}
\hline & Training set RMS error $(\mathrm{dB})$ & Validation set RMS error $(\mathrm{dB})$ & Testing set RMS error $(\mathrm{dB})$ & $\mid$ error $\mid>2 \mathrm{~dB}$ Testing $(\%)$ \\
\hline Single BPNN (nonturbulent) & 1.90 & 2.14 & 2.18 & 16.2 \\
Env. adaptive (nonturbulent) & 1.64 & 1.70 & 1.84 & 12.3 \\
Single BPNN (turbulent) & 1.17 & 1.54 & 1.55 & 5.1 \\
Env. adaptive (turbulent) & 1.08 & 1.15 & 1.36 & 3.7 \\
\hline
\end{tabular}

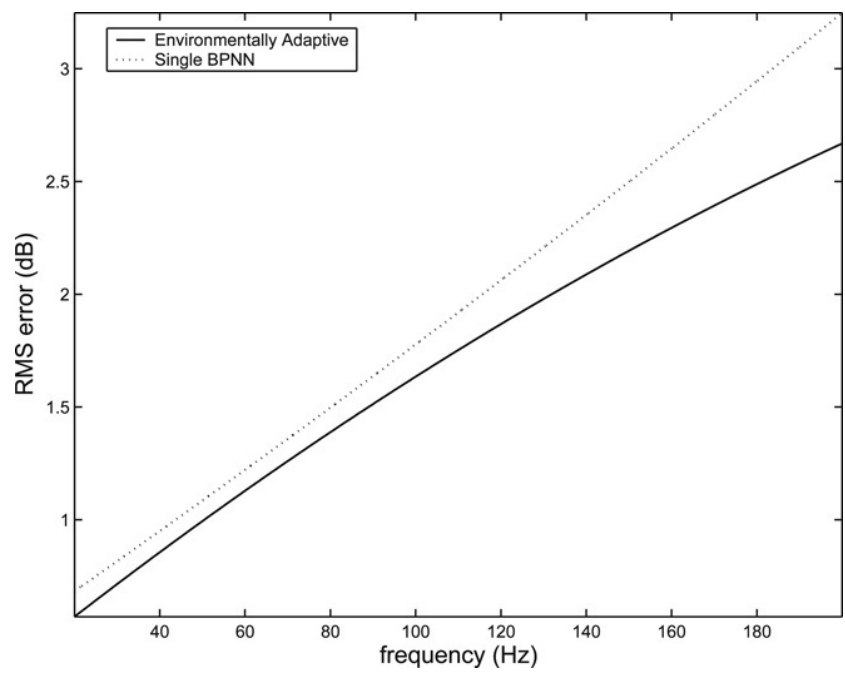

(a) Non-turbulent.

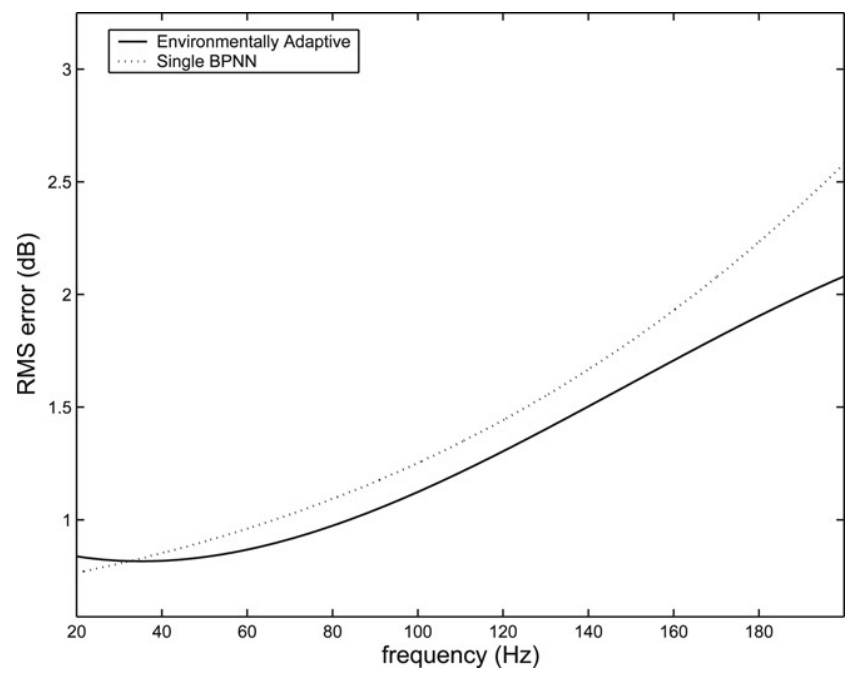

(b) Turbulent.

Fig. 4. Testing set RMS error trends for the environmentally adaptive and single BPNN predictors as a function of frequency.

when compared to nonturbulent cases with comparable input parameter combinations.

Although we have indicated the generally large reduction in execution time to obtain TL with the use of a neural network, it would be appropriate to put the accuracy of the results in Table 2 in perspective. The RMS errors that we have found in this study are well within the variability in SPL that has been observed due to existing atmospheric turbulence. That is, if experiments were conducted under actual atmospheric, ground, and geometrical conditions that were within the respective ranges of this study, the SPL would nearly always vary by amounts substantially more than $2 \mathrm{~dB}$.

We now examine the testing set RMS error trends as a function of the individual input parameters, which have significant influence on the TL. The binning procedure proposed in Mungiole and Wilson (2006) is used to generate the plots of the RMS error as a function of individual input parameters. Here all cases in the testing set were divided into 40 bins each containing an equal number of cases, based on the input parameter under investigation. The RMS error was then computed separately for each of these binned data points. A smooth curve was then fit to the binned data points, which represent the RMS error trend as a function of the input parameter of interest.

Fig. 4(a) shows the RMS error trend on the non-turbulent testing set as a function of frequency $f$, for the two prediction methodologies. As can be seen, the RMS error values are lower for the environmentally adaptive method over the entire frequency range considered in our study, and the improvements are most pronounced at high frequencies. It appears that the collaborative behaviour of the environmentally adaptive system is able to better compensate for the increased refraction that occurs at high frequencies. A similar plot for the turbulent atmospheric condition is shown in Fig. 4(b), where the environmentally adaptive system improves prediction accuracy, especially at high frequencies.

Fig. 5(a) and (b) show the testing set RMS error trend as a function of horizontal separation between source and receiver $x_{r}$ for the non-turbulent and turbulent PE model data sets, respectively. The environmentally adaptive method also led to improvements at large values of $x_{r}$, as shown in Fig. 5(a) and (b). For the environmentally adaptive method we see a levelling off of the nonturbulent RMS error curve shown in Fig. 5(a) at approximately $750 \mathrm{~m}$ up to the maximum $x_{r}$ value used in our study of $900 \mathrm{~m}$. The collaborative nature of the environmentally adaptive system provides an ability to better account for some of the PE model effects that occur at large $x_{r}$ values, such as the increased likelihood of encountering refractive shadow regions where sound levels are locally depressed.

Fig. 6(a) and (b) show the RMS error as a function of $A=c_{*} / u_{*}$, or the sound speed scale divided by the friction velocity for the nonturbulent and turbulent $\mathrm{PE}$ model data sets, respectively. Once again the environmentally adaptive method improves prediction accuracy across the entire range of $A$ values in Fig. 6(a) and (b), with slight improvements in the stable regime as $A$ becomes positive and large, both with and without atmospheric turbulence. Under stable atmospheric conditions sound waves are likely to be refracted 


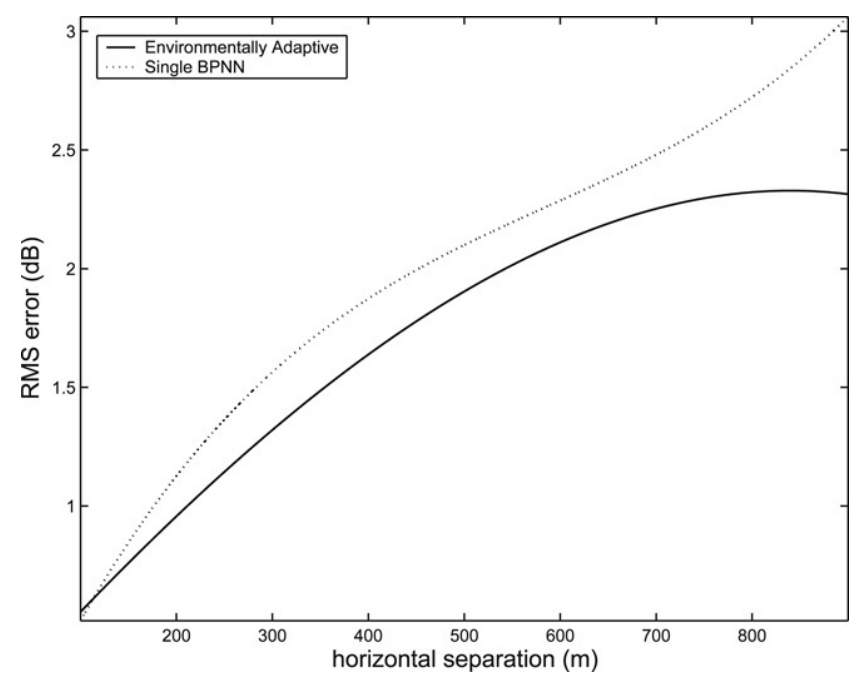

(a) Nonturbulent.

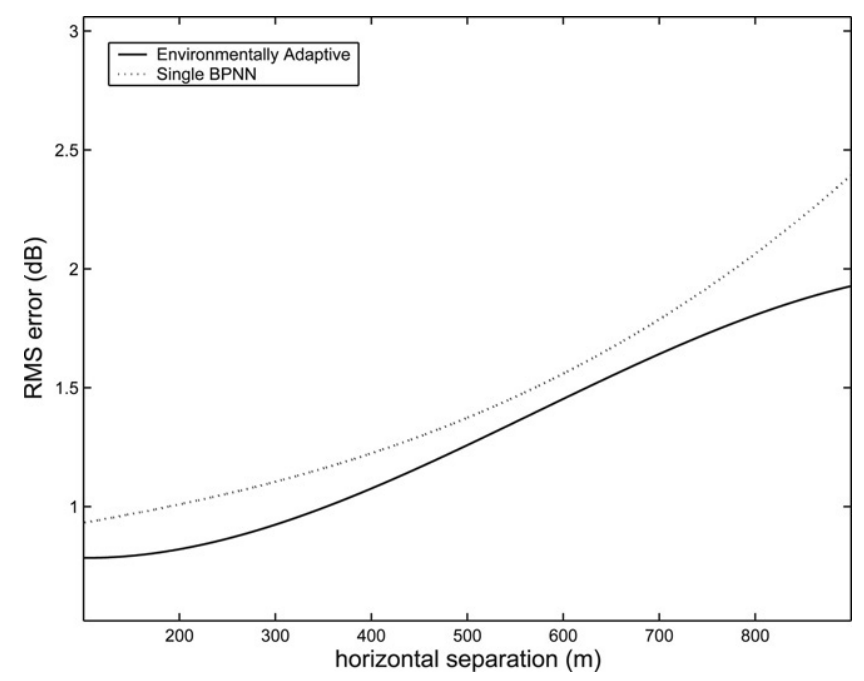

(b) Turbulent.

Fig. 5. Testing set RMS error trends for the environmentally adaptive and single BPNN predictors as a function of horizontal separation between source and receiver.

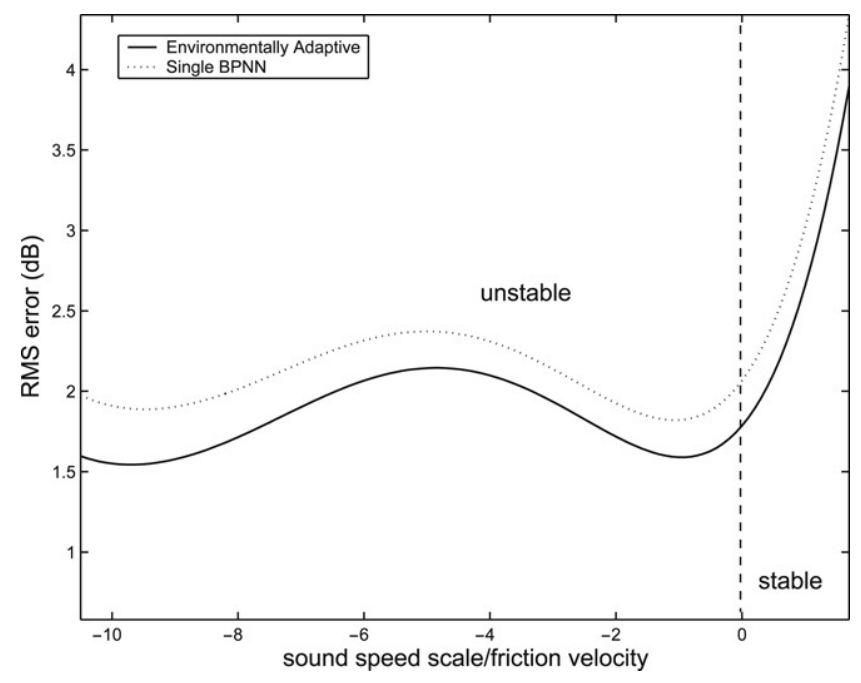

(a) Non-turbulent.

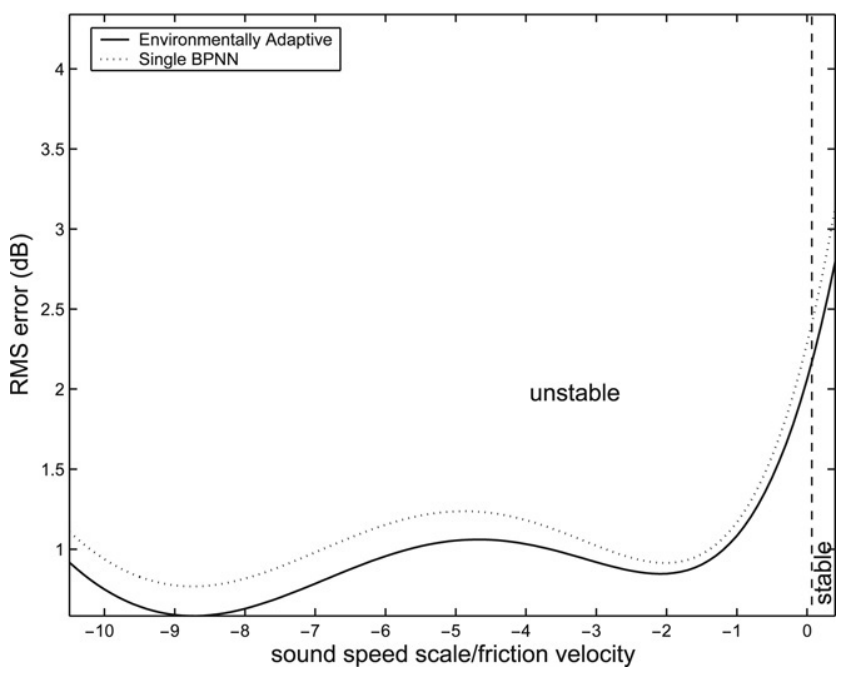

(b) Turbulent.

Fig. 6. Testing set RMS error trends for the environmentally adaptive and single BPNN predictors as a function of $A=c_{*} / u_{*}$.

downward, and it is more likely for destructive interference between direct and reflected waves to occur under these conditions. Furthermore, near ground ducting increases sound pressure levels in the stable atmospheric regime at distances where destructive interference minima are not present. These effects make fast and accurate prediction of the TL in the stable atmospheric regime a difficult problem, and are partly responsible for the large RMS errors at positive $A$ values shown in Fig. 6(a) and (b). Although, the environmentally adaptive prediction system used an environmentally specific data subset corresponding to exclusively stable regime data, the RMS error improvements in Fig. 6(a) and (b) appear minimal in the stable regime. This result suggests a possible limitation of neural network modelling for atmospherically stable acoustic environments. The environmentally adaptive method improved the RMS error trend as a function of the other input parameters from Table 1, but the results are not shown here due to space constraints. The RMS error trends as a function of the other input parameters for the single BPNN TL prediction method can be found in Mungiole and Wilson (2006), and for the environmentally adaptive TL prediction system in Wichern (2006).

Similar to the results obtained in Mungiole and Wilson (2006), the outliers in this study generally occurred for the higher frequency, greater horizontal separation, and stable atmospheric conditions. While the environmentally adaptive predictors could have been partitioned differently to reduce the number of outliers in specific ranges of these parameters, it would likely have increased the number of outliers for selected ranges of other parameter combinations. An appropriate quality control practical feature to consider in future work would be to also include the probability of specific conditions actually occurring in nature and include that as an additional weighting factor in determining the neural network. As an example, 3.7\% of the test cases are outliers for the turbulent environmentally adaptive neural network but it is possible that the parameter 
combinations comprising these outlier cases may naturally occur somewhat less than $3.7 \%$ of the time.

We now examine the ability of the environmentally adaptive and single BPNN predictors to approximate the nonturbulent PE model as horizontal separation, $x_{r}$ is varied, while all other input parameters are held constant. Fig. 7(a) and (b) show the ability of the two prediction methods in approximating the PE model at frequencies of $100 \mathrm{~Hz}$ and $150 \mathrm{~Hz}$ respectively, when all input parameters besides horizontal separation are held constant at their baseline values from Table 1. For both frequencies displayed in Fig. 7(a) and (b) the single BPNN generally seems unreliable in providing an accurate approximation of the PE model. Under baseline parameter values from Table 1 the environmentally adaptive predictor approximates the PE model almost perfectly at a frequency $100 \mathrm{~Hz}$ as shown in Fig. 7(a). As frequency is increased to a value of $150 \mathrm{~Hz}$ the environmentally adaptive predictor loses accuracy in approximating the PE model at large values of horizontal separation, although it remains more accurate than the single BPNN. At high frequencies and/or large horizontal separations the greatest attenuation of the acoustic signature is observed, and refraction and reflection effects are more likely to alter the acoustic signature, leading to the increased errors at large $x_{r}$ values in Fig. 7(b) for a frequency of $150 \mathrm{~Hz}$. Fig. 7(c) and (d) show similar plots when the other input parameters, except frequency and horizontal separation, are set to their extreme values from Table 1 . Once again we notice the inaccuracy of the single BPNN in approximating the PE model as horizontal separation is varied for all investigated frequencies. We also notice that the behaviour of the PE model is increasingly nonlinear under extreme conditions, especially at the high frequency of $150 \mathrm{~Hz}$, shown in Fig. 7(d). Although the environmentally adaptive method exhibits improved approximation accuracy over the single BPNN in Fig. 7(a)-(d), its accuracy deteriorates as the PE model behaviour becomes increasingly nonlinear at high frequencies. The extreme parameter conditions shown in Fig. 7(c) and (d), correspond to an upwind propagation direction which increases refraction, and a highly unstable atmosphere that causes sound to refract upward increasing the probability of refractive shadow regions. It is likely that these atmospheric refraction effects are responsible for the decrease in prediction accuracy at high frequencies exhibited by the neural network-based prediction systems in Fig. 7(d).

The four cases shown in Fig. 7(a)-(d), all considered an unstable or upward refracting atmospheric condition. Fig. 7(e) and (f) show the approximation capabilities of the two predictors in the stable atmospheric regime, where all input parameters besides frequency and horizontal separation are set at their baseline values from Table 1. Also, the sound speed scale, $c_{*}$, is set to the positive (stable atmospheric regime) value of 0.05 m/s. From Fig. 7(e) and (f) we see that the PE model behaviour is highly nonlinear for both frequencies shown here. We also notice in Fig. 7(e) and (f), sharp interference minima, or precipitous drops in the TL for certain combinations of horizontal separation and frequency. If turbulence were included in the PE model, these interference minima would tend to be reduced as discussed in Section 2.4. Thus, we are not overly concerned by the failure of the two prediction methods to capture these large dips, but would like to capture the near ground ducting present in the stable regime, or the unusually high TL values at large horizontal separation values in Fig. 7(e) and (f), outside of the characteristic minima. Examples of near ground ducting in the stable regime can be seen in both Figs. 7(e) and (f) at horizontal separations between approximately 600 and $900 \mathrm{~m}$. Developing prediction methodologies that capture the near ground ducting present in the stable atmospheric regime, while not being adversely affected by the characteristic minima is a problem, which necessitates further study.

As discussed in Section 2.4, the inclusion of atmospheric turbulence in the PE model tends to "smooth out" some of the large drops in the nonturbulent PE model TL curves shown in Fig. 7(d)-(f). This should then make it easier for our developed neural network-based prediction methods to approximate the turbulent PE model data set. Thus, we feel that the worst case scenario has been presented in Fig. 7(a)-(f), and similar plots are not needed for the turbulent condition.

\section{Conclusion}

In this paper an environmentally adaptive acoustic TL prediction system was developed. This environmentally adaptive system improves the accuracy of PE model approximation in terms of RMS error and number of outliers, by using a bank of expert BPNN predictors to estimate the TL. The system also exploits a decision-making component to assign fuzzy confidence measures to the appropriate experts for a given input parameter combination. The decision-making device also allows the user to input a priori information or requirements into the system, such as the likelihood and/or tactical importance of certain environmental conditions being encountered in practice. A fusion centre is then used to combine the weighted estimates from the appropriate expert predictors obtaining the final TL estimate. The environmentally adaptive prediction system was tested on a synthetic PE model acoustic data set and its results were extensively benchmarked against those of a single BPNN predictor. The results demonstrate that the environmentally adaptive system reduced the testing set RMS error by $15.6 \%$, and the number of outliers by $24.1 \%$ on the nonturbulent PE model data set, when compared with the single BPNN predictor. The environmentally adaptive system also improved TL prediction accuracy at high frequencies and large horizontal separation values.

Additionally, the proposed TL prediction system was tested on PE model data where the effects of atmospheric turbulence were also synthesized. In a turbulent atmosphere the predicted TL values exhibited better accuracy in approximating the PE model, as turbulent scattering tends to reduce the severity of acoustic shadow regions and destructive interference patterns. For example, the environmentally adaptive prediction system had a testing set RMS error of $1.36 \mathrm{~dB}$ for the turbulent condition, and $1.84 \mathrm{~dB}$ for the nonturbulent case. Moreover, the environmentally adaptive TL prediction system reduced the 


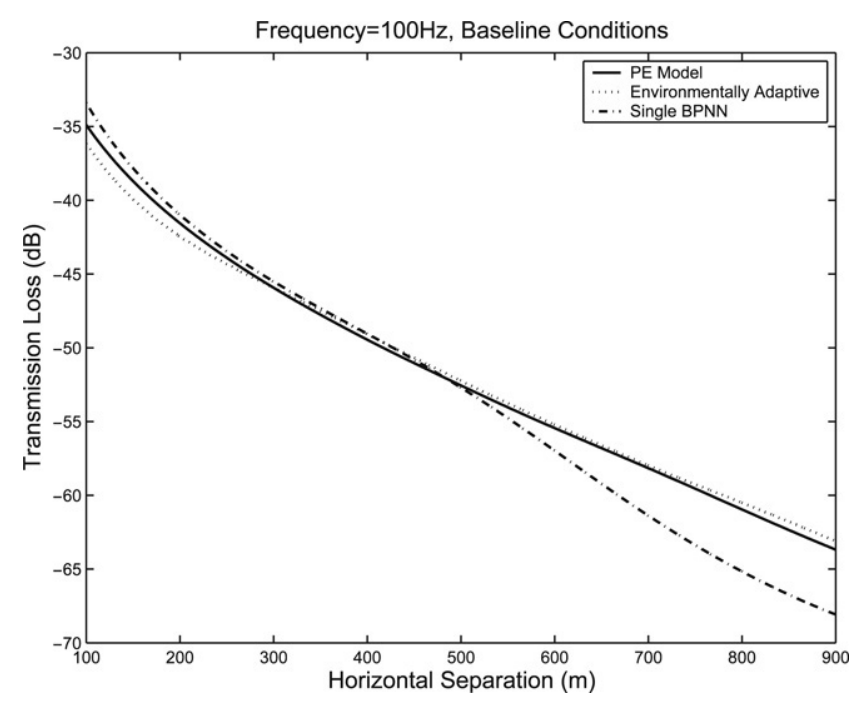

(a) Baseline, $100 \mathrm{~Hz}$.

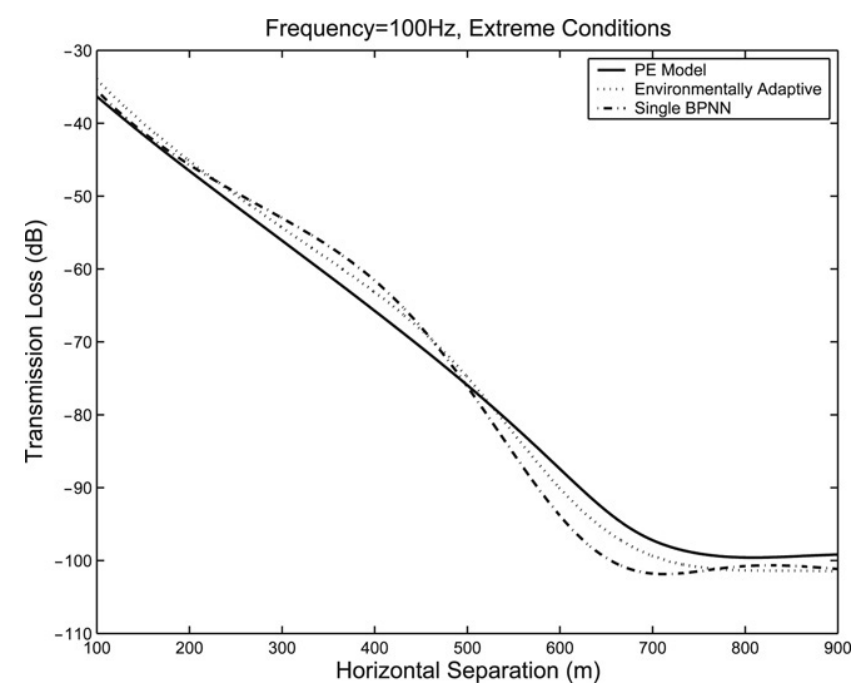

(c) Extreme, $100 \mathrm{~Hz}$.

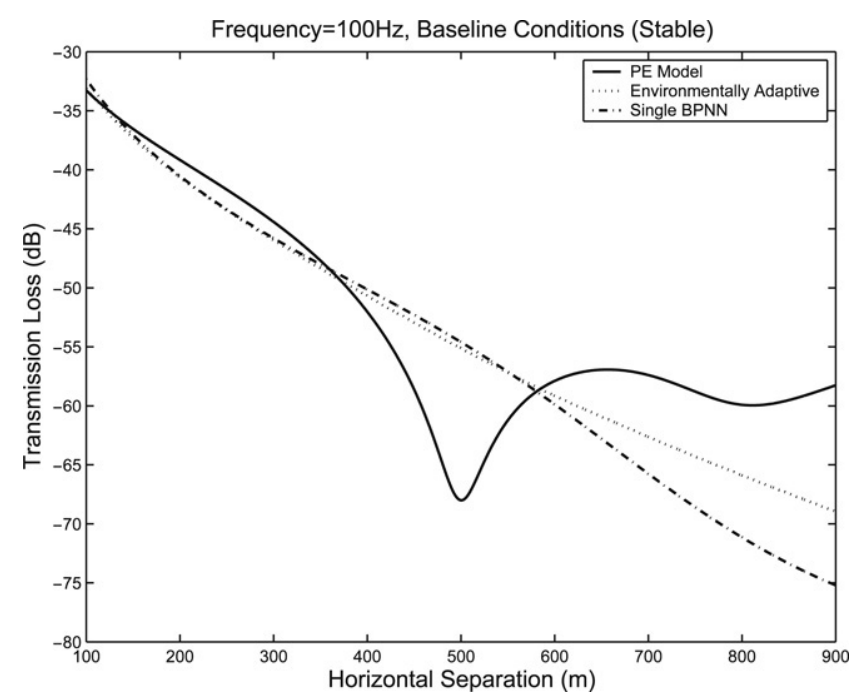

(e) Baseline (stable), $100 \mathrm{~Hz}$.

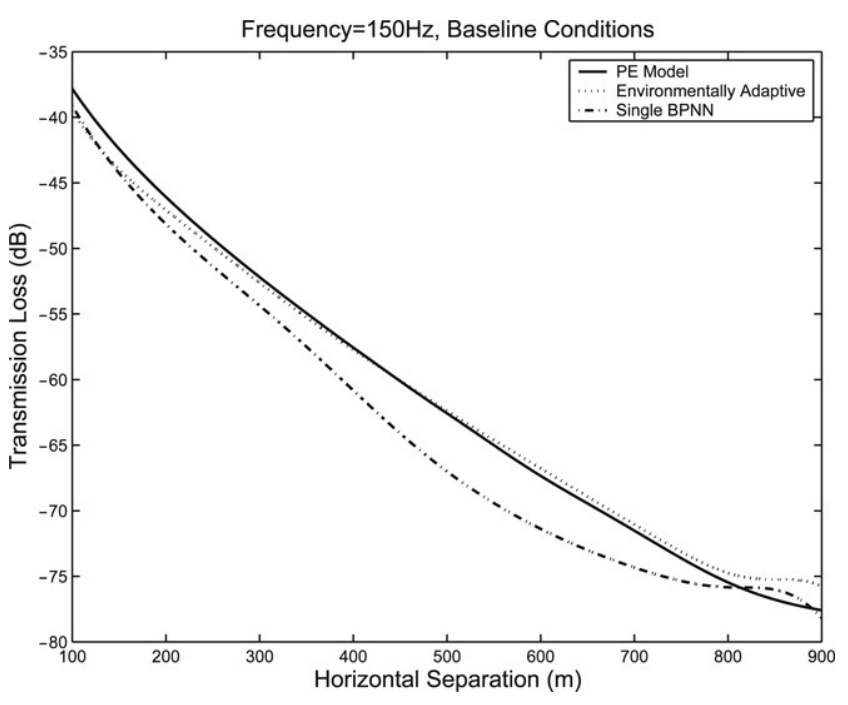

(b) Baseline, $150 \mathrm{~Hz}$.

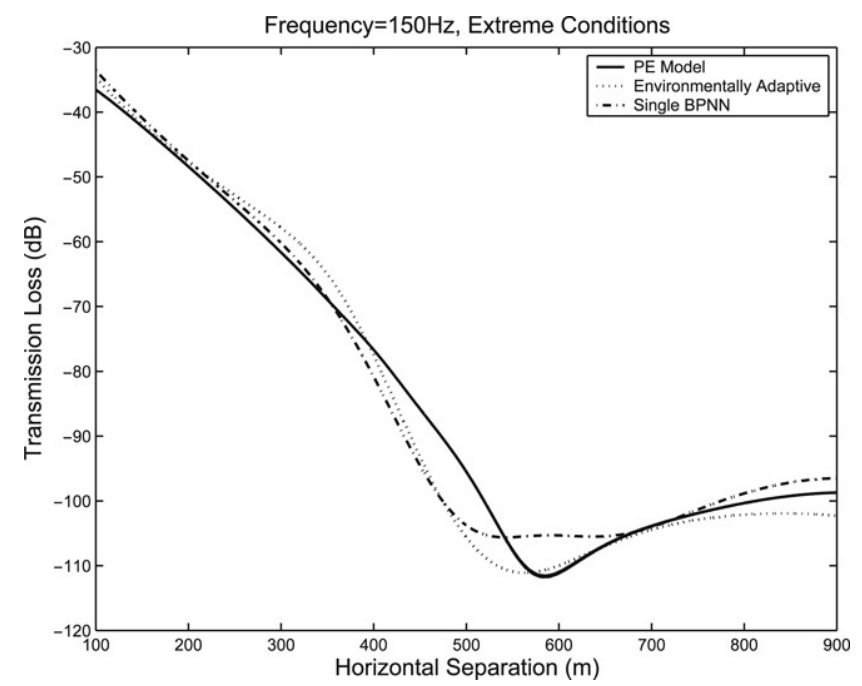

(d) Extreme, $150 \mathrm{~Hz}$.

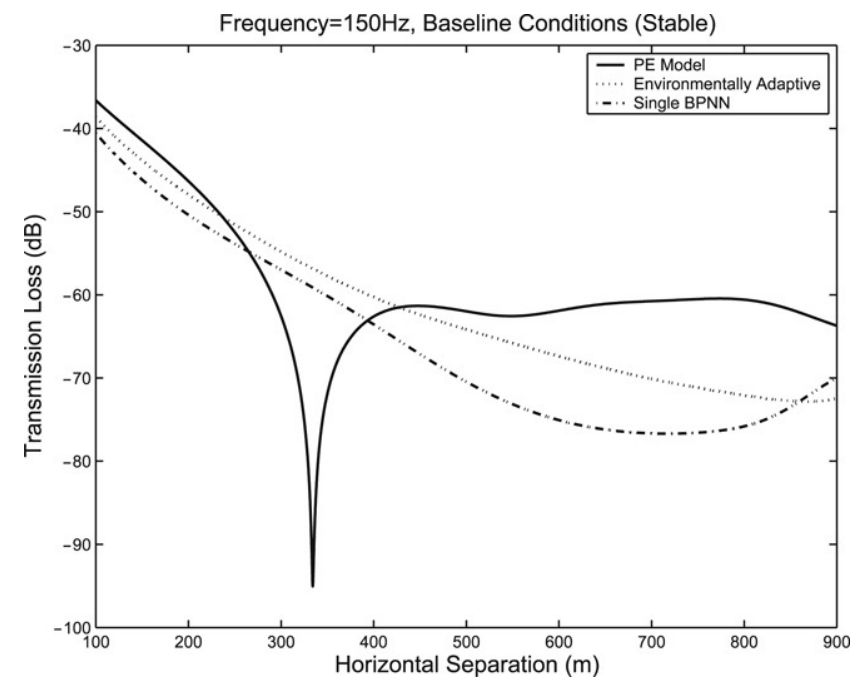

(f) Baseline (stable), $150 \mathrm{~Hz}$.

Fig. 7. Variation of the acoustic transmission loss as horizontal separation is varied for the nonturbulent PE model and two different neural network-based prediction schemes. 
percentage of testing set outliers by $27.5 \%$ when compared with single BPNN predictor for the turbulent condition.

Finally, it should be mentioned that the environmentally adaptive prediction system reduced the computational times necessary to compute a single TL value by a factor of approximately 10 when compared with the PE model in the nonturbulent condition, a frequency of $20 \mathrm{~Hz}$, and horizontal separation between source and receiver of $100 \mathrm{~m}$. At a frequency of $200 \mathrm{~Hz}$, a horizontal separation of $900 \mathrm{~m}$, and turbulent condition, computation times are reduced by a factor of roughly 50,000, which is significant for many acoustic sensing scenarios. Evaluation of the environmentally adaptive TL prediction system on acoustic data sets with increased ranges of frequency $(20-300 \mathrm{~Hz})$ and horizontal separation (100-2000 m) are currently under investigation. Computationally efficient schemes to simulate the effects of atmospheric turbulence on acoustic signatures are also being examined. Additionally, the future research should involve the design of physics-based neural network TL prediction systems that can capture mapping functions in a manner similar to multidimensional partial differential equations (PDE).

\section{Acknowledgments}

This research was supported by the DoD Centre for Geosciences/Atmospheric Research at Colorado State University via Cooperative Agreement DAAD19-02-2-0005 with the Army Research Laboratory.

\section{References}

Attenborough, K. (1985). Acoustical impedance models for outdoor ground surfaces. Journal of Sound and Vibration, 91, 521-544.

Attenborough, K., Taherzadeh, S., Bass, H. E., Di, X., Raspert, R., Becker, G. R., et al. (1995). Benchmark cases for outdoor sound propagation models. Journal of the Acoustical Society of America, 97, 173-191.

Benediktsson, O. E. J. A., Sveinsson, J. R., \& Swain, P. (1997). Parallel consensual neural network. IEEE Transactions on Neural Networks, 8, 54-64.

Chevallier, F., Chèruy, F., Scott, N. A., \& Chedin, A. (1998). A neural network approach for a fast and accurate computation of longwave radiative budget. Journal of Applied Meteorology, 37, 1385-1397.

Collier, S. L., \& Wilson, D. K. (2003). Performance bounds for passive sensor arrays operating in a turbulent medium: Plane-wave analysis. Journal of the Acoustical Society of America, 113, 2704-2918.

Collier, S. L., \& Wilson, D. K. (2004). Performance bounds for passive sensor arrays operating in a turbulent medium: Spherical-wave analysis. Journal of the Acoustical Society of America, 116, 987-1001.

Embleton, T. F. W. (1996). Tutorial on sound propagation outdoors. Journal of the Acoustical Society of America, 100, 31-48.

Gilbert, K. E., \& Di, X. (1993). A fast Green's function method for one-way sound propagation in the atmosphere. Journal of the Acoustical Society of America, 94, 2343-2352.
Hagan, M. T., \& Menhaj, M. B. (1994). Training of feedforward networks with the Marquardt algorithm. IEEE Transactions on Neural Networks, 113(6), 989-993.

Harris, C., Hong, X., \& Gan, Q. (2002). Adaptive modelling, estimation and fusion from data. New York: Springer-Verlag.

Hastie, T., Tibshirani, R., \& Friedman, J. (2001). The elements of statistical learning: Data mining, inference, and prediction. Springer-Verlag.

Haykin, S. (1999). Neural networks: A comprehensive foundation (2nd ed.). Upper Saddle River, NJ: Prentice Hall.

Klir, G. J., \& Yuan, B. (1995). Fuzzy sets and fuzzy logic: Theory and applications. Upper saddle River, NJ: Prentice Hall.

Krasnopolsky, V. M., \& Fox-Rabinovitz, M. S. (2006). A new synergetic paradigm in environmental modeling: Hybrid models combining deterministic and machine learning components. Ecological Modeling, 191(1), 5-18.

Lin, C. T., \& Lee, C. S. G. (1991). Neural-network-based fuzzy logic control and decision system. IEEE Transactions on Computers, 40(12), 1320-1336.

Lumley, J. L. (1971). Stochastic tools in turbulence. New York: Academic.

Monin, A. S., \& Obukhov, A. M. (1954). Basic laws of turbulent mixing in the atmosphere near the ground. Trudy Akademiia Nauk SSR Geofizicheskogo Instituta, 24, 163-187.

Mungiole, M., \& Wilson, D. K. (2006). Prediction of outdoor sound transmission loss with an artificial neural network. Applied Acoustics, 67, 324-345.

Mungiole, M., Wilson, D.K., \& Ostashev, V. (2004). Sound propagation classification schemes using atmospheric similarity parameters. In 11th long range sound propagation symposium.

Salomons, E. (2001). Computational atmospheric acoustics. Norwell, MA: Kluwer Academic Publishers.

Srour, N. (1999). Unattended ground sensors-A prospective for operational needs and requirements. ARL technical report prepared for NATO.

Stull, R. B. (1988). An introduction to boundary layer meteorology. Boston, MA: Kluwer.

Tang, Y., \& Hsieh, W. W. (2003). ENSO simulations and prediction in a hybrid coupled model with data assimilation. Journal of the Meteorological Society of Japan, 81, 1-19.

West, M., Gilbert, K., \& Sack, R. A. (1992). A tutorial on the Parabolic Equation (PE) model used for long range sound propagation in the atmosphere. Applied Acoustics, 37, 31-49.

West, M., Sack, R. A., \& Walden, F. (1991). The Fast Field Program (FFP). A second tutorial: Application to long range sound propagation in the atmosphere. Applied Acoustics, 33, 199-228.

Wichern, G. (2006). Adaptive methods for rapid acoustic transmission loss prediction in the atmosphere. Master's thesis. Colorado State University.

Wichern, G., Azimi-Sadjadi, M.R., \& Mungiole, M. (2006). An environmentally adaptive system for rapid acoustic transmission loss prediction. In Proc. of IEEE international joint conference on neural networks (pp. 5118-5125).

Wilson, D. K. (1997). Simple relaxational models for the acoustical properties of porous media. Applied Acoustics, 50, 171-188.

Wilson, D. K. (1998). Performance bounds for direction-of-arrival arrays operating in atmospheric turbulence. Journal of the Acoustical Society of America, 103, 1306-1319.

Wilson, D. K. (2000). A turbulence spectral model for sound propagation in the atmosphere that incorporates shear and buoyancy forcings. Journal of the Acoustical Society of America, 108, 2021-2038.

Wilson, D. K. (2003). The sound-speed gradient and refraction in the nearground atmosphere. Journal of the Acoustical Society of America, 113, $750-757$.

Zadeh, L. (1965). Fuzzy sets. Information and Control, 8, 338-353. 